\title{
Relaxation of nonlocal m-dissipative differential inclusions
}

\author{
S. Bilal, O. Cârjă, T. Donchev, N. Javaid, A. I. Lazu
}

\begin{abstract}
We show here that the set of the integral solutions of a nonlocal differential inclusion is dense in the set of the solution set of the corresponding relaxed differential inclusion. We further define a notion of limit solution and show that the set of limit solutions is closed and is the closure of the set of integral solutions. An illustrative example is provided.
\end{abstract}

\section{Introduction}

Let $X$ be a Banach space and $I=\left[t_{0}, T\right] \subset \mathbb{R}_{+}$. Consider the nonlinear differential inclusion with nonlocal initial conditions

$$
\left\{\begin{array}{l}
\dot{x}(t) \in A x(t)+F(t, x(t)), t \in I \\
x\left(t_{0}\right)=g(x(\cdot)),
\end{array}\right.
$$

where $A: D(A) \subset X \rightrightarrows X$ is an m-dissipative operator, $F: I \times X \rightrightarrows X$ is a multivalued map and $g: C(I, X) \rightarrow \overline{D(A)}$ is a given function.

A large class of partial differential equations (inclusions) can be written in the form (1.1). We refer the reader to [7], where nonlocal evolution inclusions with time delay are comprehensively studied. Among others, we cite $[8,22]$ where the problem (1.1) is studied in the case of linear $A$ and $[3,18,23]$ when $A$ is nonlinear. See also [17] for a viability result when $F$ is single valued.

Key Words: m-dissipative functional evolution inclusions, nonlocal initial conditions 2010 Mathematics Subject Classification: Primary 34B15; Secondary 34A60, 35R70. Received: 17.01.2019

Accepted: 25.02.2019 
In the present paper we study the relation between the solutions of the problem (1.1) and the solutions of the corresponding relaxed problem

$$
\left\{\begin{array}{l}
\dot{y}(t) \in A y(t)+\overline{c o} F(t, y(t)), t \in I \\
y\left(t_{0}\right)=g(y(\cdot)) .
\end{array}\right.
$$

More precisely, we prove that the solution set of (1.1) is dense in the solution set of the convexified problem (1.2). This kind of result, known in literature as relaxation theorem, is very important in the theory of differential inclusions and in the optimal control problems (see, e.g., [14, 20]). Notice that the solution set of the relaxed problem (1.2) is not closed in general. A natural question that arises here is related to the structure of its closure. In order to answer this question, we consider the limits of some approximate solutions of (1.1), called limit solutions, which are not necessarily solutions of (1.1). We prove that the closure of the solution set of (1.1) is the set of the limit solutions of (1.1).

There are several papers devoted to relaxation theorems for the local form of the inclusions (1.1) and (1.2), i.e., when the second conditions are replaced by $x\left(t_{0}\right)=x_{0}$ and $y\left(t_{0}\right)=y_{0}$ respectively, with $x_{0}, y_{0} \in \overline{D(A)}$ (see, e.g., $[9,10,11,13,20])$. A common assumption in these papers is that $A$ generates a compact semigroup. Further, in [20], the dual space $X^{*}$ is strictly convex and $F$ is Lipschitz with compact values. In [9] the dual space $X^{*}$ is uniformly convex. The relaxation theorem of [9] was extended in [11] by weakening the Lipschitz condition on the multifunction $F$ to one-sided Lipschitz. A more general form was considered in [10] assuming that the duality map of $X$ is single valued. Further, a weaker condition on the multifunction $F$ is considered in [10], namely, one-sided Perron.

To our knowledge, our relaxation result given here is the first one in the case of nonlocal conditions. We assume that the multifunction $F$ is Lipschitz continuous with closed and bounded values. However we don't assume anything about the semigroup. Therefore, our relaxation theorem is new even in the case of local initial conditions. The present paper appears to be a natural extension of [2], where the existence of solutions of (1.1) was considered.

This paper is devoted to nonlocal fully nonlinear evolution systems. We determine the closure of the solution set. To author's knowledge no related results exist in the literature. The limit solution set of (2.3) is compact in the case when $A$ generates a compact semigroup. Notice that if $F(t, x)$ is single valued and almost continuous, then every limit solution is actually solution.

\section{Preliminaries}

In this section we give some definitions and auxiliary results used in this paper. 
Let $X$ be a Banach space with the norm $|\cdot|$. For $\mathcal{A} \subset X, \overline{\mathcal{A}}$ stands for its closure and $\overline{c o} \mathcal{A}$ for its closed convex hull. The distance from a point to a set is $\operatorname{dist}(x, \mathcal{A})=\inf _{a \in \mathcal{A}}|x-a|$. Let $\mathcal{A}, \mathcal{B} \subset X$ be nonempty bounded sets. The Hausdorff-Pompeiu distance is defined by $D_{H}(\mathcal{A}, \mathcal{B})=$ $\max \{\operatorname{ex}(\mathcal{A}, \mathcal{B}), \operatorname{ex}(\mathcal{B}, \mathcal{A})\}$, where $\operatorname{ex}(\mathcal{A}, \mathcal{B})=\sup _{a \in \mathcal{A}} \operatorname{dist}(a, \mathcal{B})$. For any bounded set $\mathcal{A}$ we denote $\|\mathcal{A}\|=\sup \{|x| ; x \in \mathcal{A}\}$.

The duality map of $X, J: X \rightrightarrows X^{*}$, is defined by $J(x)=\left\{x^{*} \in\right.$ $\left.X^{*} ;\left\langle x^{*}, x\right\rangle=|x|^{2}=\left|x^{*}\right|^{2}\right\}$, where $\langle\cdot, \cdot\rangle$ is the duality pairing. Recall that if $X^{*}$ is uniformly convex then $J(\cdot)$ is single valued. For any nonempty closed bounded set $\mathcal{A} \subset X$ and $l \in X^{*}$ we define $\sigma(l, \mathcal{A})=\sup _{a \in \mathcal{A}}\langle l, a\rangle$. Recall that $\sigma(l, \mathcal{A})=\sigma(l, \overline{c o} \mathcal{A})$.

We denote by $[x, u]_{+}$the right directional derivative of the norm calculated at $x$ in the direction $u$, i.e.,

$$
[x, u]_{+}=\lim _{h \downarrow 0} \frac{|x+h u|-|x|}{h} .
$$

It is known that, when $J$ is single valued, $\langle J(x), y\rangle=|x|[x, y]_{+}$for any $x, y \in$ $X$.

The multifunction $F: I \times X \rightrightarrows X$ is called lower semicontinuous (LSC) at $(t, x) \in I \times X$ if, for any $v \in F(t, x)$ and any sequence $\left(\left(t_{n}, x_{n}\right)\right)_{n}$ with $t_{n} \rightarrow t$ and $x_{n} \rightarrow x$, there exists a sequence $\left(v_{n}\right)_{n}$ with $v_{n} \in F\left(t_{n}, x_{n}\right)$ for every $n \in \mathbb{N}$, such that $v_{n} \rightarrow v$. It is called LSC if it is LSC at every $(t, x) \in I \times X$. The multifunction $F(\cdot, \cdot)$ is called continuous if it is continuous with respect to the Hausdorff-Pompeiu distance. $F(\cdot, \cdot)$ is called almost LSC (almost continuous) if for every $\varepsilon>0$ there exists a compact interval $\Delta_{\varepsilon} \subseteq I$ with meas $\left(I \backslash \Delta_{\varepsilon}\right)<\varepsilon$ such that $F_{\mid \Delta_{\varepsilon} \times X}$ is LSC (continuous). Here, meas denotes the Lebesgue measure.

For $f \in L^{1}(I, X)$, consider the Cauchy problem

$$
\left\{\begin{array}{l}
\dot{x}(t) \in A x(t)+f(t), t \in I \\
x\left(t_{0}\right)=x_{0} \in \overline{D(A)}
\end{array}\right.
$$

In the case when $J$ is single valued, following [5], we say that $x \in C(I, X)$ is an (integral) solution of (2.1) if $x\left(t_{0}\right)=x_{0}$ and for every $u \in D(A)$ and $v \in A(u)$ the following inequality holds

$$
|x(t)-u|^{2} \leq|x(s)-u|^{2}+2 \int_{s}^{t}\langle J(x(\tau)-u), f(\tau)+v\rangle d \tau
$$

for $t_{0} \leq s \leq t \leq T$. See, e.g., [4] for the definition of the integral solution when $J$ is not necessarily single valued. 
It is well known that for each $x_{0} \in \overline{D(A)}$ the Cauchy problem (2.1) has a unique integral solution on $\left[t_{0}, T\right]$. Moreover, if $x(\cdot)$ and $y(\cdot)$ are integral solutions of $(2.1)$ with $x\left(t_{0}\right)=x_{0}$ and $y\left(t_{0}\right)=y_{0}$, then

$$
|x(t)-y(t)| \leq\left|x_{0}-y_{0}\right|+\int_{t_{0}}^{t}\left[x(s)-y(s), f_{x}(s)-f_{y}(s)\right]_{+} d s
$$

for every $t \in\left[t_{0}, T\right]$ (see, e.g., [16]). In particular,

$$
|x(t)-y(t)| \leq\left|x_{0}-y_{0}\right|+\int_{t_{0}}^{t}\left|f_{x}(s)-f_{y}(s)\right| d s,
$$

for every $t \in\left[t_{0}, T\right]$.

Consider now the differential inclusion

$$
\left\{\begin{array}{l}
\dot{x}(t) \in A x(t)+F(t, x(t)), t \in I \\
x\left(t_{0}\right)=x_{0},
\end{array}\right.
$$

where $x_{0} \in \overline{D(A)}$. We say that $x \in C(I, X)$ is a solution of (2.3) if there exists $f_{x} \in L^{1}(I, X)$ with $f_{x}(t) \in F(t, x(t))$ a.e. on $I$, such that $x(\cdot)$ is an integral solution of (2.1). We say that $x \in C(I, X)$ is a solution of (1.1) if it is a solution of $(2.3)$ and $x\left(t_{0}\right)=g(x(\cdot))$.

We refer the reader to $[6,14]$ for the theory of m-dissipative differential inclusions and to [4] for some recent trends.

The function $f_{x}$ involved above is called pseudoderivative of $x(\cdot)$.

\section{The main result}

In this section we will prove the main result of the present paper, that is, the density of the solution set of (1.1) into the solution set of (1.2).

We first introduce the standing hypotheses $(\mathbf{H})$ :

(h1) There exists a Lebesgue integrable function $\kappa(\cdot)$ such that $\|F(t, 0)\| \leq$ $\kappa(t)$ for any $t \in I$.

(h2) There exists a Lebesque integrable function $L(\cdot)$ such that $D_{H}(F(t, x), F(t, y)) \leq L(t)|x-y|$ for any $t \in I$ and any $x, y \in X$.

(h3) The multifunction $F$ is almost continuous with nonempty closed values.

(h4) The function $g: C(I, X) \rightarrow \overline{D(A)}$ satisfies $|g(x)-g(y)| \leq K\|x-y\|_{\infty}$ for some $K>0$ and for any $x, y \in C(I, X)$. We denoted by $\|\cdot\|_{\infty}$ the sup-norm of $C(I, X)$. 
(h5) $K \exp \left(\int_{t_{0}}^{T} L(s) d s\right)<1$.

Remark 3.1. Notice that from (h1) and (h2) it follows that

$$
\|\overline{c o} F(t, x)\| \leq \kappa(t)+L(t)|x|
$$

for any $(t, x) \in I \times X$.

To simplify the presentation, in what follows we denote $l(t):=\int_{t_{0}}^{t} L(s) d s$ and, for any $\delta \geq 0, \beta(\delta):=(\exp (l(T))+\delta) K$.

The following result will be used later in the paper.

Lemma 3.2. [2, Theorem 2.3] Assume that the multifunction $F$ satisfies (h1)-(h3). Then, for every $\varepsilon>0$ and $\delta>0$, every $x_{0}, y_{0} \in \overline{D(A)}$ and every solution $x(\cdot)$ of $(2.3)$ there exists a solution $y(\cdot)$ of

$$
\left\{\begin{array}{l}
\dot{y}(t) \in A y(t)+F(t, y(t)) \\
y\left(t_{0}\right)=y_{0}
\end{array}\right.
$$

such that

$$
|x(t)-y(t)| \leq(\exp (l(t))+\varepsilon)\left|x_{0}-y_{0}\right|
$$

and

$$
\left|f_{x}(t)-f_{y}(t)\right| \leq L(t)(\exp (l(t))+\varepsilon)\left|x_{0}-y_{0}\right|+\delta
$$

for any $t \in I$, where $f_{x}(\cdot)$ and $f_{y}(\cdot)$ are pseudoderivatives of $x(\cdot)$ and $y(\cdot)$, respectively.

The following result is analogue of the well-known Filippov-Plis lemma. See, e.g., [12].

Lemma 3.3. Assume that $F$ satisfies (h1) $-(\mathbf{h} 3)$. Let $\varepsilon>0$ and $y_{0} \in \overline{D(A)}$. Let $y(\cdot)$ be a solution of

$$
\left\{\begin{array}{l}
\dot{y}(t) \in A y(t)+F(t, y(t)+\varepsilon \mathbb{B}) \\
y\left(t_{0}\right)=y_{0}
\end{array}\right.
$$

Then, for every $\mu>0$, there exists a solution $z(\cdot)$ of

$$
\left\{\begin{array}{l}
\dot{z}(t) \in A z(t)+F(t, z(t)) \\
z\left(t_{0}\right)=y_{0}
\end{array}\right.
$$

such that $|y(t)-z(t)| \leq \varepsilon \exp (l(t))+\mu$ for all $t \in I$. 
Proof. Let $\left(\delta_{n}\right)_{n}$ be a decreasing sequence of positive numbers such that the series $\sum_{k=0}^{\infty} \delta_{k}$ is convergent. Let $f_{y}(\cdot)$ be a pseudoderivative of the solution $y(\cdot)$. Since $f_{y}(t) \in F(t, x(t)+\varepsilon \mathbb{B})$, then $\operatorname{dist}\left(f_{y}(t), F(t, y(t))\right) \leq L(t) \varepsilon$. Due to (h2) and (h3), for $\delta_{0}>0$ there exists $f_{0}(t) \in F(t, y(t))$ such that $\left|f_{y}(t)-f_{0}(t)\right| \leq$ $L(t) \varepsilon+\frac{\delta_{0}}{T-t_{0}}$. Let $y^{0}(\cdot)$ be the solution of

$$
\left\{\begin{array}{l}
\dot{x}(t) \in A x(t)+f_{0}(t), \\
x\left(t_{0}\right)=y_{0} .
\end{array}\right.
$$

Then $\left|y^{0}(t)-y(t)\right| \leq \int_{t_{0}}^{t}\left|f_{0}(s)-f_{y}(s)\right| d s \leq \varepsilon l(t)+\delta_{0}$.

There exists a strongly measurable selection $f_{1}(t) \in F\left(t, y^{0}(t)\right)$ such that

$$
\left|f_{1}(t)-f_{0}(t)\right| \leq L(t)\left|y^{0}(t)-y(t)\right|+\frac{\delta_{1}}{T-t_{0}} .
$$

Let $y^{1}(\cdot)$ be the solution of

$$
\left\{\begin{array}{l}
\dot{y}^{1}(t) \in A y^{1}(t)+f_{1}(t) \\
y^{1}\left(t_{0}\right)=y_{0}
\end{array}\right.
$$

Then

$$
\left|y^{1}(t)-y^{0}(t)\right| \leq \int_{t_{0}}^{t}\left|f_{1}(s)-f_{0}(s)\right| d s \leq \int_{t_{0}}^{t} L(s)\left(l(s) \varepsilon+\delta_{0}\right) d s+\delta_{1} .
$$

Then $\left|y^{1}(t)-y^{0}(t)\right| \leq \frac{l^{2}(t)}{2 !} \varepsilon+\delta_{0} l(t)+\delta_{1}$. We have used the fact that, for any natural $k$,

$$
\int_{t_{0}}^{t} L(s) l^{k}(s) d s=\frac{l^{k+1}(t)}{k+1} .
$$

There exists a strongly measurable function $f_{2}(t) \in F\left(t, y^{1}(t)\right)$ such that

$$
\left|f_{2}(t)-f_{1}(t)\right| \leq L(t)\left|y^{1}(t)-y^{0}(t)\right|+\frac{\delta_{2}}{T-t_{0}} .
$$

Let $y^{2}(\cdot)$ be the solution of

$$
\left\{\begin{array}{l}
\dot{y}^{2}(t) \in A y^{2}(t)+f_{2}(t) \\
y^{2}\left(t_{0}\right)=y_{0}
\end{array}\right.
$$


After trivial calculations one derives

$$
\left|y^{2}(t)-y^{1}(t)\right| \leq \frac{l^{3}(t)}{3 !} \varepsilon+\frac{l^{2}(t)}{2 !} \delta_{0}+l(t) \delta_{1}+\delta_{2}
$$

One can continue by induction and get a sequence $\left(y^{n}(\cdot)\right)_{n}$ satisfying

$$
\left|y^{n}(t)-y^{n-1}(t)\right| \leq \frac{l^{n+1}(t)}{(n+1) !} \varepsilon+\sum_{j=0}^{n} \frac{l^{j}(t)}{j !} \delta_{n-j} \leq \frac{l^{n+1}(T)}{(n+1) !} \varepsilon+\sum_{j=0}^{n} \frac{l^{j}(T)}{j !} \delta_{n-j}
$$

for any $t \in I$ and a sequence $\left(f_{n}(\cdot)\right)_{n}$ satisfying $f_{n}(t) \in F\left(t, y^{n-1}(t)\right)$ a.e. on $I$ and

$$
\left|f_{n}(t)-f_{n-1}(t)\right| \leq L(t)\left|y^{n-1}(t)-y^{n-2}(t)\right|+\frac{\delta_{n}}{T-t_{0}}
$$

for all $t \in I$.

Since the series $\sum_{k=0}^{\infty} \frac{l^{k}(T)}{k !}$ and $\sum_{k=0}^{\infty} \delta_{k}$ are convergent, then also is the se$\operatorname{ries} \sum_{k=1}^{\infty} \sum_{j=0}^{k} \frac{l^{j}(T)}{j !} \delta_{k-j}$. Therefore, the sequence $\left(y_{n}(\cdot)\right)_{n}$ is Cauchy, hence it is uniformly convergent so some continuous function $z(\cdot)$. In a similar way one can prove that $\left(f_{n}(\cdot)\right)_{n}$ converges strongly in $L^{1}(I, X)$ to some function $f_{z}(\cdot)$. It is standard to prove that $z(\cdot)$ is a solution of $(3.3), f_{z}(\cdot)$ being its pseudoderivative.

Furthermore,

$$
|z(t)-y(t)| \leq \varepsilon \sum_{k=1}^{\infty} \frac{l^{k}(t)}{k !}+\sum_{k=0}^{\infty} \sum_{j=0}^{k} \frac{l^{j}(t)}{j !} \delta_{k-j}=\exp (l(t))\left(\varepsilon+\sum_{k=0}^{\infty} \delta_{k}\right) .
$$

The proof is completed.

Theorem 3.4. Assume $\mathbf{( H )}$. Then, for any $\varepsilon>0$, any $x_{0} \in \overline{D(A)}$ and any solution $x(\cdot)$ of $(2.3)$ there exists a solution $z(\cdot)$ of

$$
\left\{\begin{array}{l}
\dot{z}(t) \in A z(t)+F(t, z(t)) \\
z\left(t_{0}\right)=g(z(\cdot))
\end{array}\right.
$$

such that $\|z(\cdot)-x(\cdot)\|_{\infty} \leq \frac{\left|x_{0}-g(x(\cdot))\right|}{K(1-\beta(0))}+\varepsilon$.

Proof. Let $\varepsilon>0, x(\cdot)$ a solution of $(2.3)$ and $f_{x}(\cdot)$ the corresponding pseudoderivative of $x(\cdot)$. Let $\delta \in\left(0, \frac{1-\beta(0)}{K}\right)$ so that $\beta(\delta)<1$. 
Due to Lemma 3.2, there exists a solution $y^{1}(\cdot)$ of $(3.2)$ with $y_{0}:=g(x(\cdot))$ such that, for any $t \in I,\left|y^{1}(t)-x(t)\right| \leq(\exp (l(t))+\delta)\left|x_{0}-y_{0}\right|$ and $\mid f_{x}(t)-$ $f_{1}(t)|\leq L(t)(\exp (l(t))+\delta)| x_{0}-y_{0} \mid+\delta$. Here, $f_{1}(\cdot)$ is the pseudoderivative of $y^{1}(\cdot)$.

Applying again Lemma 3.2 we get a solution $y^{2}(\cdot)$ of $(3.2)$ with $y\left(t_{0}\right)=$ $y_{1}:=g\left(y^{1}(\cdot)\right)$ such that $\left|y^{2}(t)-y^{1}(t)\right| \leq(\exp (l(t))+\delta)\left|y_{1}-y_{0}\right|$ and $\mid f_{2}(t)-$ $f_{1}(t)|\leq L(t)(\exp (l(t))+\delta)| y_{1}-y_{0} \mid+\delta / 2$ for $t \in I$. We denoted by $f_{2}(\cdot)$ the pseudoderivative of $y^{2}(\cdot)$. Using (h4) and the previous estimates, we have that

$\left|y^{2}(t)-y^{1}(t)\right| \leq K(\exp (l(t))+\delta)\left\|y^{1}(\cdot)-x(\cdot)\right\|_{\infty} \leq K(\exp (l(T))+\delta)^{2}\left|x_{0}-y_{0}\right|$

and

$$
\left|f_{2}(t)-f_{1}(t)\right| \leq L(t) K(\exp (l(T))+\delta)^{2}\left|x_{0}-y_{0}\right|+\frac{\delta}{2}
$$

for $t \in I$. We continue by induction and define a sequence $\left(y^{n}(\cdot)\right)_{n}$ in $C(I, X)$ in the following way. If $y^{n}(\cdot)$ is given for $n \geq 1$, then we define $y^{n+1}(\cdot)$ as the solution of (3.2), with $y_{1}$ replaced by $y_{n}:=g\left(y^{n}(\cdot)\right)$, given by Lemma 3.2. Then

$$
\left|y^{n+1}(t)-y^{n}(t)\right| \leq K^{n}(\exp (l(T))+\delta)^{n+1}\left|x_{0}-y_{0}\right|=\frac{1}{K} \beta(\delta)^{n+1}\left|x_{0}-y_{0}\right|
$$

and

$$
\begin{gathered}
\left|f_{n+1}(t)-f_{n}(t)\right| \leq L(t) K^{n}\left(\exp (l(T)+\delta)^{n+1}\left|x_{0}-y_{0}\right|+\frac{\delta}{2^{n}}\right. \\
=\frac{1}{K} L(t) \beta(\delta)^{n+1}\left|x_{0}-y_{0}\right|+\frac{\delta}{2^{n}}
\end{gathered}
$$

for $t \in I$. Since $\beta(\delta)<1$, the sequence $\left(y^{n}(\cdot)\right)_{n}$ is Cauchy, hence it converges uniformly to a continuous function $z(\cdot)$. Moreover, the corresponding sequences of pseudoderivatives $\left(f_{n}(\cdot)\right)_{n}$ converges strongly w.r.t. $L^{1}(I, X)$ to some function $f_{z}(\cdot)$. One can prove that $f_{z}(\cdot)$ is the pseudoderivative of $z(\cdot)$, $f_{z}(t) \in F(t, z(t))$ a.e. on $I$ and $z\left(t_{0}\right)=g(z(\cdot))$, i.e., $z(\cdot)$ is a solution of (3.4). Furthermore, taking $y^{0}(t):=x(t)$, we have that

$$
\begin{aligned}
|z(t)-x(t)| \leq \sum_{n=0}^{\infty}|| y^{n+1}(\cdot) & -y^{n}(\cdot)||_{\infty} \leq \sum_{n=0}^{\infty} \frac{1}{K} \beta(\delta)^{n+1}\left|x_{0}-g(x(\cdot))\right| \\
= & \frac{\left|x_{0}-g(x(\cdot))\right|}{K(1-\beta(\delta))} .
\end{aligned}
$$

Then, for $\delta$ small enough,

$$
|z(t)-x(t)| \leq \frac{\left|x_{0}-g(x(\cdot))\right|}{K(1-\beta(0))}+\varepsilon
$$

for any $t \in I$. 
Now we will study the closure of the solution set of the problem (1.1). To this end we introduce the notion of limit solution of (1.1).

Definition 3.5. A continuous function $x(\cdot)$ is said to be a limit solution of (1.1) if there exist two sequences of positive numbers $\left(\varepsilon_{n}\right)_{n}$ and $\left(\delta_{n}\right)_{n}$ decreasing to zero and a sequence $\left(y^{n}(\cdot)\right)_{n}$ in $C(I, X)$ such that, for any $n \geq 1, y^{n}(\cdot)$ is a solution of

$$
\left\{\begin{array}{l}
\dot{y}(t) \in A y(t)+F\left(t, y(t)+\varepsilon_{n} \mathbb{B}\right) \\
y\left(t_{0}\right)=y_{n}
\end{array}\right.
$$

where $\left(y_{n}\right)_{n} \subset \overline{D(A)}$ satisfies $\left|y_{n}-g\left(y^{n}(\cdot)\right)\right|<\delta_{n}$ and $\lim _{n \rightarrow \infty} y^{n}(t)=x(t)$ uniformly on $I$.

Notice that, in general, the limit solutions have no pseudoderivatives.

Theorem 3.6. Under the hypotheses $\mathbf{( H )}$, the solution set of (1.1) is dense in the set of limit solutions of (1.1). Moreover, the set of limit solutions of (1.1) is closed.

Proof. Let $x(\cdot)$ be a limit solution of $(1.1)$ and let $\left(\varepsilon_{n}\right)_{n},\left(\delta_{n}\right)_{n}$ and $\left(y^{n}(\cdot)\right)_{n}$ be the corresponding sequences given by Definition 3.5.

For every $n \geq 1$, due to Lemma 3.3 , there exists a solution $z^{n}(\cdot)$ of

$$
\left\{\begin{array}{l}
\dot{z}(t) \in A z(t)+F(t, z(t)) \\
z\left(t_{0}\right)=y^{n}\left(t_{0}\right)
\end{array}\right.
$$

satisfying

$$
\left\|z^{n}(\cdot)-y^{n}(\cdot)\right\|_{\infty} \leq \varepsilon_{n} \exp (l(T))+\varepsilon_{n} .
$$

Now, due to Theorem 3.4, for every $n \geq 1$, there exists a solution $u^{n}(\cdot)$ of

$$
\left\{\begin{array}{l}
\dot{u}(t) \in A u(t)+F(t, u(t)) \\
u\left(t_{0}\right)=g(u(\cdot))
\end{array}\right.
$$

such that

$$
\left\|u^{n}(\cdot)-z^{n}(\cdot)\right\|_{\infty} \leq \frac{\left|g\left(z^{n}(\cdot)\right)-z^{n}\left(t_{0}\right)\right|}{K(1-\beta(0))}+\varepsilon_{n} .
$$

Further, since $\left|g\left(z^{n}(\cdot)\right)-z^{n}\left(t_{0}\right)\right| \leq K\left\|z^{n}(\cdot)-y^{n}(\cdot)\right\|_{\infty}+\delta_{n}$, by (3.5), we get that

$$
\left\|u^{n}(\cdot)-z^{n}(\cdot)\right\|_{\infty} \leq\left[K \varepsilon_{n}(\exp (l(T))+1)+\delta_{n}\right] \frac{1}{K(1-\beta(0))}+\varepsilon_{n} .
$$

Finally, the above estimates lead to the fact that $x(t)=\lim _{n \rightarrow \infty} u^{n}(t)$ uniformly on I.

The fact that the set of limit solutions is closed is trivial. 
Notice that Theorem 3.6 has been proved in general Banach spaces.

Let us now state the main result of this paper.

Theorem 3.7. Let $X$ be such that its duality map $J(\cdot)$ is single valued and assume $(\mathbf{H})$. Then the solution set of (1.1) is dense in the solution set of $(1.2)$.

Before proving the result, we recall that under the hypotheses of Theorem 3.7 the problem (1.1) has at least one solution, as it was proved in [2].

Proof. Let $x(\cdot)$ be a solution of (1.2). Then, $x\left(t_{0}\right)=g(x(\cdot))$ and there exists $f_{x}(\cdot) \in L^{1}(I, X)$ with $f_{x}(t) \in \overline{c o} F(t, x(t))$ a.e. on $I$ such that $x(\cdot)$ is an integral solution of

$$
\left\{\begin{array}{l}
\dot{y}(t) \in A y(t)+f_{x}(t), t \in I \\
y\left(t_{0}\right)=x\left(t_{0}\right)
\end{array}\right.
$$

Fix $\mu>0$. We will give the proof in several steps.

I) First, we define a submultifunction of $F$, almost LSC with nonempty closed bounded values, that will be used in the second step of the proof to construct an approximate solution of (1.1) which starts from $x\left(t_{0}\right)$ and remains close to $x(\cdot)$ on $I$.

To this end, let $\varepsilon \in(0, \mu)$ and fix $0<\delta<\varepsilon$. We define the multifunction $G_{\delta}: I \times \overline{D(A)} \rightrightarrows X$ by

$$
G_{\delta}(t, y)=\left\{\begin{array}{l}
\left\{v \in F(t, y) ;\left\langle J(x(t)-y), f_{x}(t)-v\right\rangle<\right. \\
(L(t)|x(t)-y|+\delta)|x(t)-y|\}, \quad \text { if }|x(t)-y| \geq \delta \\
F(t, y), \quad \text { if }|x(t)-y|<\delta .
\end{array}\right.
$$

We claim that $\bar{G}_{\delta}(\cdot, \cdot)$ is almost LSC with nonempty closed bounded values.

Let $(t, y) \in I \times \overline{D(A)}$. If $|x(t)-y|<\delta$ then, clearly $G_{\delta}(t, y) \neq \emptyset$. Consider the case when $|x(t)-y| \geq \delta$. Since $f_{x}(t) \in \overline{c o} F(t, x(t))$ and $\sigma(l, \overline{c o} A)=$ $\sigma(l, A)$ for any bounded set $A \subset X$ and any $l \in X^{*}$, we have that

$$
\begin{gathered}
\left\langle J(x(t)-y), f_{x}(t)\right\rangle \leq \sigma(J(x(t)-y), \overline{c o} F(t, x(t)))=\sigma(J(x(t)-y), F(t, x(t))) \\
=\sup _{w \in F(t, x(t))}\langle J(x(t)-y), w\rangle .
\end{gathered}
$$

Therefore, for every $\xi>0$ there exists $g \in F(t, x(t))$ such that

$$
\left\langle J(x(t)-y), f_{x}(t)-g\right\rangle<\xi|x(t)-y| .
$$


It is well known that $\sigma(J(x(t)-y), F(t, x(t)))-\sigma(J(x(t)-y), F(t, y)) \leq$ $|x(t)-y| D_{H}(F(t, x(t)), F(t, y))$. Further, using hypothesis (h2), there exists $v \in F(t, y)$ such that

$$
\langle J(x(t)-y), g-v\rangle \leq L(t)|x(t)-y|^{2}+\xi|x(t)-y| .
$$

Due to (3.6) and (3.7) we get that

$$
\begin{array}{r}
\quad\left\langle J(x(t)-y), f_{x}(t)-v\right\rangle \leq\langle J(x(t)-y), g-v\rangle+\xi|x(t)-y| \\
\leq L(t)|x(t)-y|^{2}+\xi|x(t)-y|=|x(t)-y|(L(t)|x(t)-y|+\xi) .
\end{array}
$$

Here $\xi>0$ is arbitrary, hence $G_{\delta}(t, y)$ is nonempty.

Now we will prove that $G_{\delta}(\cdot, \cdot)$ is almost LSC. Consequently, $\bar{G}_{\delta}(\cdot, \cdot)$ is also almost LSC. Clearly, $G_{\delta}(\cdot, \cdot)$ is almost LSC on $\{(t, y) \in I \times \overline{D(A)} ;|x(t)-y|<$ $\delta\}$.

Since $F(\cdot, \cdot)$ is almost continuous, $f_{x}(\cdot)$ is strongly measurable and $L(\cdot)$ is measurable, for any $\mu>0$ there exists a compact set $I_{\mu} \subset I$ with meas $\left(I \backslash I_{\mu}\right)<$ $\mu$ such that $\left.F\right|_{I_{\mu} \times X}$ is continuous and $\left.f_{x}\right|_{I_{\mu}},\left.L\right|_{I_{\mu}}$ are continuous functions. Let $(\bar{t}, \bar{y}) \in I_{\mu} \times \overline{D(A)}$ be such that $|x(\bar{t})-\bar{y}| \geq \delta$. Let $\bar{v} \in G_{\delta}(\bar{t}, \bar{y})$ and $\left(\left(t_{k}, y_{k}\right)\right)_{k} \subset I_{\mu} \times \overline{D(A)}$ with $t_{k} \rightarrow \bar{t}$ and $y_{k} \rightarrow \bar{y}$.

Since $\left\langle J(x(\bar{t})-\bar{y}), f_{x}(\bar{t})-\bar{v}\right\rangle=|x(\bar{t})-\bar{y}|\left[x(\bar{t})-\bar{y}, f_{x}(\bar{t})-\bar{v}\right]_{+}$and $\bar{v} \in G_{\delta}(\bar{t}, \bar{y})$, we obtain that $\left[x(\bar{t})-\bar{y}, f_{x}(\bar{t})-\bar{v}\right]_{+} \leq L(\bar{t})|x(\bar{t})-\bar{y}|+\delta-\gamma$, for some $\gamma>0$.

There exists a sequence $\left(v_{k}\right)_{k}$ with $v_{k} \in F\left(t_{k}, y_{k}\right)$ for any natural $k$ such that $v_{k} \rightarrow \bar{v}$. As $[\cdot, \cdot]_{+}$is upper semicontinuous as a real valued function and $L(\cdot)$ is continuous at $\bar{t}$, there exists $\bar{k} \in \mathbb{N}$ such that $\left[x(\bar{t})-\bar{y}, f_{x}(\bar{t})-\bar{v}\right]_{+} \geq$ $\left[x\left(t_{k}\right)-y_{k}, f_{x}\left(t_{k}\right)-v_{k}\right]_{+}-\gamma / 2$ and $|L(\bar{t})| x(\bar{t})-\bar{y}\left|-L\left(t_{k}\right)\right| x\left(t_{k}\right)-y_{k}||<\gamma / 2$, for any $k \geq \bar{k}$. Hence, $\left[x\left(t_{k}\right)-y_{k}, f_{x}\left(t_{k}\right)-v_{k}\right]_{+}<L\left(t_{k}\right)\left|x\left(t_{k}\right)-y_{k}\right|+\delta$, for any $k \geq \bar{k}$. It follows that

$$
\begin{gathered}
\left\langle J\left(x\left(t_{k}\right)-y_{k}\right), f_{x}\left(t_{k}\right)-v_{k}\right\rangle=\left|x\left(t_{k}\right)-y_{k}\right|\left[x\left(t_{k}\right)-y_{k}, f_{x}\left(t_{k}\right)-v_{k}\right]_{+} \\
\leq\left|x\left(t_{k}\right)-y_{k}\right|\left(L\left(t_{k}\right)\left|x\left(t_{k}\right)-y_{k}\right|+\delta\right),
\end{gathered}
$$

i.e., $v_{k} \in G_{\delta}\left(t_{k}, y_{k}\right)$ for any $k \geq \bar{k}$. Thus, $G_{\delta}(\cdot, \cdot)$ is $\operatorname{LSC}$ at $(\bar{t}, \bar{y})$.

II) Now, using the submultifunction $G_{\delta}$ defined in the first step of the proof, we provide a continuous function $y(\cdot)$, solution of

$$
\left\{\begin{array}{l}
\dot{y}(t) \in A y(t)+F(t, y(t)+\varepsilon \mathbb{B}), t \in I \\
y\left(t_{0}\right)=x\left(t_{0}\right),
\end{array}\right.
$$

such that $\|x-y\|_{\infty} \leq \varepsilon$. More precisely, $y(\cdot)$ is the solution of

$$
\left\{\begin{array}{l}
\dot{y}(t) \in A y(t)+f_{y}(t), t \in I \\
y\left(t_{0}\right)=x\left(t_{0}\right)
\end{array}\right.
$$


for some function $f_{y} \in L^{1}(I, X)$ with $f_{y}(t) \in F(t, y(t)+\varepsilon \mathbb{B})$ a.e. on $I$ satisfying $\operatorname{dist}\left(f_{y}(t), \bar{G}_{\delta}(t, y(t))\right) \leq \mu_{\delta}(t)$ for any $t \in I, \mu_{\delta}(\cdot)$ being a Lebesgue integrable function with

$$
\int_{I} \mu_{\delta}(t) d t \leq \delta
$$

First, let us remark that, if $z(\cdot)$ is a solution of

$$
\left\{\begin{array}{l}
\dot{z}(t) \in A z(t)+f_{z}(t) \\
f_{z}(t) \in \overline{c o} F(t, z(t)+\mathbb{B}) \\
z\left(t_{0}\right)=x\left(t_{0}\right)
\end{array}\right.
$$

it follows from (3.1) that $\left|f_{z}(t)\right| \leq \kappa(t)+L(t)(|z(t)|+1)$ a.e. on $I$. We mention that $\overline{c o} F(t, z+\mathbb{B})=\bigcup_{b \in \mathbb{B}} \overline{c o} F(t, z+b)$. It is standard to show with the help of Gronwall's inequality that there exists a Lebesgue integrable function $\lambda(\cdot)$ (not depending on $z(\cdot)$ ) such that $\|\overline{c o} F(t, z(t)+\mathbb{B})\| \leq \lambda(t)$ for any $t \in I$.

Let $\eta<\frac{\delta}{2\left(T-t_{0}\right)+1}$. Since $\lambda(\cdot)$ is Lebesgue integrable, there exists $\nu=$ $\nu(\delta)$ such that $\int_{J} \lambda(t) d t \leq \delta / 4$ for every measurable $J \subset I$ with meas $(J)<\nu$. Since $\bar{G}_{\delta}(\cdot, \cdot)$ is almost LSC, there exists an open set $I_{\nu} \subset I$ with meas $\left(I_{\nu}\right)<\nu$ such that $\left.\bar{G}_{\delta}\right|_{\left(I \backslash I_{\nu}\right) \times \overline{D(A)}}$ is LSC. Clearly $I_{\nu}$ is an union of a countable system of pairwise disjoint open intervals, i.e., $I_{\nu}=\bigcup_{k=1}^{\infty}\left(a_{k}, b_{k}\right)$.

Let $f_{0}(\cdot) \in L^{1}(I, X)$ be such that $f_{0}(t) \in \bar{G}_{\delta}\left(t, x_{0}\right)$ a.e. on $I$, where $x_{0}:=x\left(t_{0}\right)$, and let $y^{0}(\cdot)$ be the solution of the problem

$$
\left\{\begin{array}{l}
\dot{y}(t) \in A y(t)+f_{0}(t) \\
y\left(t_{0}\right)=x_{0}
\end{array}\right.
$$

Two cases are possible:

(i) $t_{0}=a_{0}$; in this case we take $t_{1}:=\bar{b}_{0}>t_{0}$ to be such that $\left|y^{0}(t)-x_{0}\right| \leq \varepsilon$ for $t \in\left[t_{0}, t_{1}\right]$ and $\bar{b}_{0} \leq b_{0}$.

(ii) $t_{0} \in I \backslash I_{\nu}$; in this case, since $\bar{G}_{\delta}(\cdot, \cdot)$ is $\operatorname{LSC}$ on $\left(I \backslash I_{\nu}\right) \times \overline{D(A)}$, there exists $\tilde{t}>t_{0}$ such that $\operatorname{dist}\left(f_{0}(t), G_{\delta}\left(t, y^{0}(t)\right)\right) \leq \eta$ on $\left[t_{0}, \tilde{t}\right] \bigcap\left(I \backslash I_{\nu}\right)$. We take $t_{1}$ to be the supremum of $\tilde{t}$ with the above property and such that $\left|y^{0}(t)-x_{0}\right| \leq \varepsilon$ for $t \in\left[t_{0}, \tilde{t}\right]$.

Now we define $y_{1}:=y^{0}\left(t_{1}\right) \in \overline{D(A)}$ and take $f_{1}(\cdot) \in L^{1}(I, X)$ such that $f_{1}(t) \in \bar{G}_{\delta}\left(t, y_{1}\right)$ a.e. on $I$. Let $y^{1}(\cdot)$ be the solution of

$$
\left\{\begin{array}{l}
\dot{y}(t) \in A y(t)+f_{1}(t) \\
y\left(t_{1}\right)=y_{1}
\end{array}\right.
$$


We define $t_{2}$ to be the supremum of $\tilde{t}>t_{1}$ such that $\operatorname{dist}\left(f_{1}(t), \bar{G}_{\delta}\left(t, y^{1}(t)\right)\right) \leq$ $\eta$ on $\left[t_{1}, \tilde{t}\right] \cap\left(I \backslash I_{\nu}\right)$ and $\left|y^{1}(t)-y^{1}\left(t_{1}\right)\right| \leq \varepsilon$ for $t \in\left[t_{1}, \tilde{t}\right]$.

We set $f_{y}(t)=f_{0}(t)$ on $\left[t_{0}, t_{1}\right]$ and $f_{y}(t)=f_{1}(t)$ on $\left[t_{1}, t_{2}\right]$ and define $y(\cdot)$ by $y(t)=y^{0}(t)$ on $\left[t_{0}, t_{1}\right]$ and $y(t)=y^{1}(t)$ on $\left[t_{1}, t_{2}\right]$. Clearly, $\left|f_{y}(t)\right| \leq \lambda(t)$ for $t \in\left[t_{0}, t_{2}\right]$ and $y(\cdot)$ is the solution of

$$
\left\{\begin{array}{l}
\dot{y}(t) \in A y(t)+f_{y}(t) \\
y\left(t_{0}\right)=x_{0}
\end{array}\right.
$$

on $\left[t_{0}, t_{2}\right]$ and satisfies

$$
\operatorname{dist}\left(f_{y}(t), \bar{G}_{\delta}(t, y(t))\right) \leq \eta
$$

for $t \in\left[t_{0}, t_{2}\right] \bigcap\left(I \backslash I_{\nu}\right)$.

Suppose that the solution $y(\cdot)$ of $(3.9)$ is defined on $\left[t_{0}, \tau\right), \tau<T$, and satisfies (3.10) on $\left[t_{0}, \tau\right) \cap\left(I \backslash I_{\nu}\right)$ and $\left|f_{y}(t)\right| \leq \lambda(t)$ for $t \in\left[t_{0}, \tau\right)$. We require, moreover, $f_{y}(t) \in F(t, y(t)+\varepsilon \mathbb{B})$ up to the end of the proof.

If $\tau \in\left[a_{k}, b_{k}\right)$ then $y(\cdot)$ can be extended on $\left[t_{0}, b_{k}\right]$, since $\left[\tau, b_{k}\right] \subset I_{\nu}$. We denote $\hbar=b_{k}>\tau$.

Suppose that $\tau$ is right dense. Since $\left|f_{y}(t)\right| \leq \lambda(t)$ for any $t \in\left[t_{0}, \tau\right)$, there exists $\lim _{t \uparrow \tau} y(t)=y_{\tau}$. We consider the problem

$$
\left\{\begin{array}{l}
\dot{y}(t) \in A y(t)+f_{\tau}(t) \\
y(\tau)=y_{\tau}
\end{array}\right.
$$

with the solution $y^{\tau}(\cdot)$. Here $f_{\tau}(t) \in \bar{G}_{\delta}\left(t, y_{\tau}\right)$ a.e. on $I$. Since $\tau$ is right dense, there exists $\hbar>\tau$ such that $\operatorname{dist}\left(f_{\tau}(t), \bar{G}_{\delta}\left(t, y^{\tau}(t)\right)\right) \leq \eta$ on $[\tau, \hbar] \cap\left(I \backslash I_{\nu}\right)$. We define $f_{y}(t)=f_{\tau}(t)$ on $[\tau, \hbar]$ and extend $y(\cdot)$ on $[\tau, \hbar]$ by taking $y(t)=y^{\tau}(t)$. Since $y(\cdot)$ can be extended on $\left[t_{0}, \tau+\delta\right]$ for some $\delta>0$, when $\tau<T$, one has that it can be defined on $\left[t_{0}, T\right]$.

Now we pick

$$
\mu_{\delta}(t)= \begin{cases}\eta & t \in I \backslash I_{\nu} \\ 2 \lambda(t) & t \in I_{\nu}\end{cases}
$$

It is easy to see that $\mu_{\delta}(\cdot)$ satisfies (3.8). Moreover, $\operatorname{dist}\left(f_{y}(t), \bar{G}_{\delta}(t, y(t))\right) \leq$ $\mu_{\delta}(t)$ for any $t \in I$. Then there exists a strongly measurable function $\bar{f}_{y}(\cdot)$ such that $\bar{f}_{y}(t) \in \bar{G}_{\delta}(t, y(t))$ and $\left|f_{y}(t)-\bar{f}_{y}(t)\right|<\operatorname{dist}\left(f_{y}(t), \bar{G}_{\delta}(t, y(t))\right)+\delta<$ $\mu_{\delta}(t)+\delta$ for any $t \in I$. We have either $|x(t)-y(t)|<\delta$ or $\left[x(t)-y(t), f_{x}(t)-\right.$ $\left.\bar{f}_{y}(t)\right]_{+} \leq L(t)|x(t)-y(t)|+\delta$. In the last case, from the properties of $[\cdot, \cdot]_{+}$it follows that

$$
\begin{array}{r}
{\left[x(t)-y(t), f_{x}(t)-f_{y}(t)\right]_{+} \leq L(t)|x(t)-y(t)|+\delta+\left|\bar{f}_{y}(t)-f_{y}(t)\right|} \\
\leq L(t)|x(t)-y(t)|+2 \delta+\mu_{\delta}(t) .
\end{array}
$$


Due to (2.2), we have that $|x(t)-y(t)| \leq m(t)$ for any $t \in I$, where $m(t)<\delta$ or $m(\cdot)$ is the solution of

$$
\left\{\begin{array}{l}
\dot{m}(t)=L(t) m(t)+2 \delta+\mu_{\delta}(t) \\
m\left(t_{0}\right)=0
\end{array}\right.
$$

Clearly $m(t) \leq r(t)$ for any $t \in I$, where $r(\cdot)$ is the solution of

$$
\left\{\begin{array}{l}
\dot{r}(t)=L(t) r(t)+2 \delta+\mu_{\delta}(t) \\
r\left(t_{0}\right)=\delta .
\end{array}\right.
$$

Thus, $|x(t)-y(t)| \leq r(t)$ for any $t \in I$. Furthermore, for any $t \in I$,

$$
r(t) \leq \exp \left(\int_{t_{0}}^{t} L(s) d s\right)\left(3 \delta\left(t-t_{0}\right)+\int_{t_{0}}^{t} \mu_{\delta}(s) d s\right) .
$$

Therefore, $|x(t)-y(t)| \leq(3 T+1) \delta \exp \left(\int_{t_{0}}^{T} L(t) d t\right)$ for any $t \in I$. Evidently, $\|x-y\|_{\infty} \leq \varepsilon$ for sufficiently small $\delta$. Due to (h4), we obtain that $\left|x_{0}-y_{0}\right| \leq$ $K \varepsilon$, where $y_{0}:=g(y(\cdot))$.

Finally, applying Lemma 3.3 and Theorem 3.4 we get the conclusion.

\section{Example}

In this section we give an example inspired from [15, Section 5] to illustrate the applicability of our results.

Let $\Omega \subset \mathbb{R}^{n}$ be a domain with smooth boundary $\partial \Omega$ and Lebesgue measure $\mu(\Omega)$. Let $T, S>0$ and $t_{1} \in(0, T)$. Let $\Delta_{x}$ be the usual Laplace operator.

We consider the following system

$$
\left(\begin{array}{l}
u_{t}(t, x) \\
v_{t}(t, y)
\end{array}\right) \in\left(\begin{array}{c}
\Delta_{x} u(t, x)-\partial \varphi(u(t, x)) \\
v_{y}(t, y)
\end{array}\right)+G(t, u, v)
$$

for $t \in(0, T), x \in \Omega, y \in(0, S)$, with

$$
\left\{\begin{array}{l}
\frac{\partial u}{\partial n}(t, x) \in \partial \psi(u(t, x)), t \in(0, T), x \in \partial \Omega \\
u(0, x)=\int_{\Omega} \int_{0}^{T} h(s, x, \lambda, u(s, \lambda)) d s d \lambda, x \in \Omega \\
v(t, 0)=v(t, S)=0, \quad t \in(0, T) \\
v(0, y)=\alpha_{1} v\left(t_{1}, y\right)+\alpha_{2} v(T, y), y \in(0, S)
\end{array}\right.
$$


Here $\varphi: \mathbb{R} \rightarrow \mathbb{R}$ is a proper, lower semicontinuous, convex function, with $\varphi(0)=0, \psi: \mathbb{R} \rightarrow \mathbb{R}$ is a convex, continuous function, with $0 \leq \psi(t) \leq$ $C\left(1+t^{2}\right), t \in \mathbb{R}$, for some constant $C>0$. Furthermore, $\alpha_{1}$ and $\alpha_{2}$ are real numbers.

We assume that $G:[0, T] \times \mathbb{R} \times \mathbb{R} \rightrightarrows \mathbb{R}^{2}$ is a multifunction with closed bounded values and $h:[0, T] \times \Omega \times \Omega \times \mathbb{R} \rightarrow \mathbb{R}$ is a given function.

Let $X=L^{2}(\Omega) \times L^{2}(0, S)$ endowed with the norm

$|(u, v)|_{X}=\sqrt{|u|_{L^{2}(\Omega)}^{2}+|v|_{L^{2}([0, S])}^{2}}$. Following [15], we define

$$
\begin{aligned}
& \Phi(v)=\int_{\Omega} \varphi(v(x)) d x \\
& \Psi(v)= \begin{cases}\frac{1}{2} \int_{\Omega} \mid \nabla\left(\left.v(x)\right|^{2} d x+\int_{\partial \Omega} \psi(v(x)) d s, v \in H^{1}(\Omega)\right. \\
+\infty & \text { otherwise. }\end{cases}
\end{aligned}
$$

Then $\Phi$ and $\Psi$ are proper, lower semicontinuous, convex functions, with the domains $D(\Phi)=\left\{v \in L^{2}(\Omega) ; \varphi \circ v \in L^{1}(\Omega)\right\}$ and $D(\Psi)=H^{1}(\Omega)$. Moreover, $f \in \partial \Phi(v)$ if and only if $v, f \in L^{2}(\Omega), f(x) \in \partial \varphi(v(x))$ for a.e. $x \in \Omega$ and $g \in \partial \Psi(v)$ if and only if $-\Delta v=g$ in $L^{2}(\Omega)$ and $\frac{\partial v}{\partial n}+\partial \psi(v) \ni 0$ in $L^{2}(\partial \Omega)$ (see [19, Examples 2.B and 2.E, pages 163-164]). We suppose that $\varphi$ is such that $\varphi \circ v \in L^{1}(\Omega)$ for any $v \in L^{2}(\Omega)$. By [19, Example 2.F, page 167] we have that $\partial \Phi+\partial \Psi$ is m-dissipative and equal to $\partial(\Phi+\Psi)$. Let $B=\partial(\Phi+\Psi)$. Then $D(B)=H^{1}(\Omega)$.

We define also $C: D(C) \subset L^{2}(0, S) \rightarrow L^{2}(0, S)$ by $C z=\dot{z}$ with the domain

$$
D(C)=\left\{z \in L^{2}(0, S) ; \dot{z} \in L^{2}(0, S), z(0)=z(S)=0\right\} .
$$

Clearly, $C$ defines a $C_{0}$-semigroup $\{T(t) ; t \geq 0\}$ as $T(t) z(s)=z(t+s)$ (see, e.g., [1]). It remains to show that $C$ is m-dissipative, which due to zero boundary conditions trivially follows from integrating by part. Consequently, the operator $A:=(B, C)$ is also m-dissipative. Furthermore, $\overline{D(A)}=X$.

Then, the system (4.1)-(4.2) can be rewritten in the abstract form (1.1) with $A$ as above,

$$
g(u(\cdot), v(\cdot))(x, y)=\left(\int_{\Omega} \int_{0}^{T} h(s, x, \lambda, u(s)(\lambda)) d s d \lambda, \alpha_{1} v\left(t_{1}, y\right)+\alpha_{2} v(T, y)\right)
$$

for $(u, v) \in C([0, T], X), x \in \Omega, y \in(0, S)$ and $F(t, u, v)=\left\{\left(z_{1}(\cdot), z_{2}(\cdot)\right) \in\right.$ $X ;\left(z_{1}(x), z_{2}(y)\right) \in G(t, u(x), v(y))$ for a.e. $\left.x \in \Omega, y \in(0, S)\right\}$ for $t \in[0, T]$, $u \in L^{2}(\Omega)$ and $v \in L^{2}(0, S)$.

We assume the following hypotheses. 
(G) The multifunction $G$ satisfies the following conditions:

(i) it has nonempty closed values;

(ii) $G(\cdot, u, v)$ is measurable;

(iii) $\|G(\cdot, u, v)\|$ is Lebesgue integrable;

(iv) there exists a Lebesgue integrable function $L(\cdot)$ such that

$$
D_{H}\left(G\left(t, z_{1}\right), G\left(t, z_{2}\right)\right) \leq L(t)\left|z_{1}-z_{2}\right|
$$

for any $t \in[0, T]$ and any $z_{i}=\left(u_{i}, v_{i}\right) \in \mathbb{R}^{2}, i=1,2$.

(h) The function $h$ satisfies:

(i) $h(t, x, \lambda, r)$ is measurable in $(t, x, \lambda)$ for all $r \in \mathbb{R}$;

(ii) there exist a function $H(\cdot) \in C\left(\Omega, \mathbb{R}_{+}\right)$and a positive Lebesgue integrable function $\nu(\cdot)$ such that $|h(t, x, \lambda, r)| \leq \nu(t) H(\lambda)$ for any $(t, x, \lambda, r) \in[0, T] \times$ $\Omega \times \Omega \times \mathbb{R}$

(iii) for any $(t, x, \lambda, u),(t, x, \lambda, v) \in[0, T] \times \Omega \times \Omega \times \mathbb{R}$ we have that

$$
|h(t, x, \lambda, u)-h(t, x, \lambda, v)| \leq \frac{K}{T \mu(\Omega)}|u-v| .
$$

In view of hypothesis (G), the multifunction $F$ satisfies (h1)-(h3). Recall that in any separable space any multifunction $G(t, x, y)$ with compact values, measurable in $t$ and continuous in $(x, y)$ is almost continuous (see [21]). From (h) it follows that $g(\cdot, \cdot)$ is well defined and

$$
\left|g\left(u_{1}, v_{1}\right)-g\left(u_{2}, v_{2}\right)\right|_{L^{2}(\Omega) \times L^{2}(0, S)} \leq K\left\|u_{1}-u_{2}\right\|_{\infty}+\left(\left|\alpha_{1}\right|+\left|\alpha_{2}\right|\right)\left|v_{1}-v_{2}\right|,
$$

for any $u_{1}, u_{2} \in C\left([0, T], L^{2}(\Omega)\right), v_{1}, v_{2} \in C\left([0, T], L^{2}(0, S)\right)$.

Consider the convexification of (4.1), i.e.,

$$
\left(\begin{array}{l}
u_{t}(t, x) \\
v_{t}(t, y)
\end{array}\right) \in\left(\begin{array}{c}
\Delta_{x} u(t, x)-\partial \varphi(u(t, x)) \\
v_{y}(t, y)
\end{array}\right)+\overline{c o} G(t, u, v)
$$

for $t \in(0, T), x \in \Omega, y \in(0, S)$. Then, due to Theorem 3.7 and taking into account that (4.3)-(4.2) corresponds to the system (1.2), we have the following result.

Theorem 4.1. Under the assumptions $(\mathbf{G})$ and $(\mathbf{h})$, the nonlocal problem (4.1)-(4.2) has a solution, and moreover its solution set is dense in the solution set of (4.3)-(4.2) when

$$
\left(K+\left|\alpha_{1}\right|+\left|\alpha_{2}\right|\right) \exp \left(\int_{0}^{T} L(s) d s\right)<1
$$




\section{Acknowledgement}

The work of A. I. Lazu was supported by a research grant of the TUIASI, project number TUIASI-GI-2018-0868.

\section{References}

[1] R. P. Agarwal, Asma, V. Lupulescu, D. O'Regan, Fractional semilinear equations with causal operators. RASCAM 111 (2017), 257-269.

[2] R. Ahmed, T. Donchev, A. I. Lazu, Nonlocal m-dissipative evolution inclusions in general Banach spaces. Mediterr. J. Math. 14:215 (2017), doi:10-1007/s00009-017-1016-5.

[3] S. Aizicovici, V. Staicu, Multivalued evolution equations with nonlocal initial conditions in Banach spaces. NoDEA Nonlinear Differential Equations Appl. 14 (2007), 361-376.

[4] V. Barbu, Nonlinear differential equations of monotone types in Banach spaces. Springer, New York, 2010.

[5] P. Benilan, Solutions intégrales d'équations d'évolution dans un espace de Banach. C. R. Acad. Sci. Paris Sér. A-B 274 (1972), 47-50.

[6] D. Bothe, Nonlinear Evolutions in Banach Spaces. Habilitationsschritt, Paderborn, 1999.

[7] M. Burlică, M. Necula, D. Roşu, I. I. Vrabie, Delay Differential Evolutions Subjected to Nonlocal Initial Conditions. Monographs and Research Notes in Mathematics. CRC Press, New York, 2016.

[8] L. Byszewski, Theorem about the existence and uniqueness of a semilinear nonlocal Cauchy problem. J. Math. Anal. Appl. 162 (1991), 494-505.

[9] T. Cardinali, N. Papageorgiou, F. Papalini, On nonconvex functional evolution inclusions involving m-dissipative operators. Czech. Math. J. 47 (1997), 135-148. 
[10] O. Cârjă, T. Donchev, V. Postolache, Relaxation results for nonlinear evolution inclusions with one-sided Perron right-hand side. Set-Valued Var. Anal. 22 (4), (2014), 657-671.

[11] T. Donchev, Multi-valued perturbations of $m$-dissipative differential inclusions in uniformly convex spaces. New Zealand Journal of Mathematics 31 (2002), 19-32.

[12] T. Donchev, E. Farkhi, On the theorem of Filippov-Plis and some applications. Control Cybern 38 (2009), 1-21.

[13] Y. Dong, Relaxation theorem for evolution differential inclusions. J. Math. Anal. Appl. 237 (1999), 188-200.

[14] S. Hu, N. Papageorgiou, Handbook of Multivalued Analysis. Volume II: Applications. Kluwer, Dordrecht, 2000.

[15] T. Ke, Cauchy problems for functional evolution inclusions involving accretive operators. EJQTDE 75 (2013), 1-13.

[16] V. Lakshmikantham, S. Leela, Nonlinear differential equations in abstract spaces. Pergamon Press, Oxford, 1981.

[17] M. Necula, M. Popescu, I. I. Vrabie, Viability for delay evolution equations with nonlocal initial conditions. Nonlinear Anal. 121 (2015), 164-172.

[18] A. Paicu, I. I. Vrabie, A class of nonlinear evolution equations subjected to nonlocal initial conditions. Nonlinear Anal. 72 (11) (2010), 4091-4100.

[19] R. Showalter, Monotone Operators in Banach Space and Nonlinear Partial Differential Equations. Math. Surv. Monographs 49, AMS, 1997.

[20] A. Tolstonogov, Properties of integral solutions of differential inclusions with m-accretive operators. Mat. Zametki 49 (1991), 119-131.

[21] A. Tolstonogov, Differential inclusions in a Banach space. Kluwer Academic Publishers, Dordrecht, 2000. 
[22] X. Xue, Semilinear nonlocal differential equations with measure of noncompactness in Banach spaces. J. Nanjing. Univ. Math. Big. 24 (2007), 264-276.

[23] L. Zhu, Q. Huang, G. Li, Existence and asymptotic properties of solutions of nonlinear multivalued differential inclusions with nonlocal conditions. J. Math. Anal. Appl. 390 (2012), 523-534.

S. BILAL,

Abdus Salam School of Mathematical Sciences,

68-B, New Muslim Town, Lahore, Pakistan.

Email: shams2013sms@gmail.com

O. CÂRJĂ,

Department of Mathematics,

"Al. I. Cuza" University,

Iaşi 700506 and

"Octav Mayer" Mathematics Institute,

Romanian Academy,

Iaşi 700505, Romania.

Email: ocarja@uaic.ro

T. DONCHEV,

Abdus Salam School of Mathematical Sciences, 68-B, New Muslim Town, Lahore, Pakistan.

Email: tzankodd@gmail.com

N. JAVAID,

Abdus Salam School of Mathematical Sciences, 68-B, New Muslim Town, Lahore, Pakistan.

Email: nasir.jav7000@gmail.com

A. I. LAZU,

Department of Mathematics,

"Gh. Asachi" Technical University,

Iaşi 700506, Romania.

Email: vieru_alina@yahoo.com 